Revista de Psicología de la PUCP. Vol. XX, 2, 2002

\title{
La insatisfacción corporal como variable explicativa de los trastornos alimenticios ${ }^{1}$
}

\author{
Carmen Maganto Mateo ${ }^{2}$ y Soledad Cruz Saez ${ }^{3}$ \\ Universidad del País Vasco
}

La ansiedad con relación a la comida, el temor a aumentar de peso y la obsesión por la delgadez van asociadas a variables de edad, sexo, nivel social, índice de masa corporal, distorsión e insatisfacción con la imagen corporal y se consideran factores de riesgo de los trastornos alimenticios. Se investigan y analizan las relaciones entre dichos factores así como su valor predictivo. Los participantes fueron 200 adolescentes de 14 a 18 años (104 chicos y 96 chicas). Se administraron el Cuestionario de Ansiedad Estado/Rasgo (STAI), el Inventario de Trastornos de la conducta Alimentaria (EDI-2) el Eating Attitudes Test (EAT) y dos instrumentos en construcción. Los resultados revelaron que la percepción distorsionada de la imagen del propio cuerpo, vinculada a la insatisfacción, se halla a la base de estos trastornos. La edad más sensible para las chicas está entre los 15 y 16 años y los factores predictivos encontrados son: insatisfacción con el aspecto físico global, distorsión del tamaño del cuerpo, edad y ansiedad rasgo.

Palabras clave: imagen corporal, factores de riesgo, trastornos de alimentación, adolescencia, STAI, EDI-2, EAT.

Body dissatisfaction as an explanatory variable of eating disorders

Eating disorders have increased over the last few years, as well as the age range of their initial onset which includes younger people. Food-related anxiety, fear of gaining weight, and obsession with thinness are associated with variables of age, sex, social level, body mass index, distortion and dissatisfaction with body image are considered risk factors for eating disorders. This research examined the relationship among these factors and analyzed their predictive value. The participants were 200 adolescents ( 104 boys and 96 girls), aged between 14 and 17 years. The tests used were the STAI, EDI-2, EAT and two experimental tests. The results indicated that underlying these disorders were distorted body-image perception and dissatisfaction. The risk for girls increased between 15 and 16 years. Dissatisfaction with global physical aspect, distortion of body size, age, and trait anxiety were revealed as predictive factors.

Key words: body image, risk factors, eating disorders, adolescence STAI, EDI-2, EAT.

Esta investigación ha sido posible gracias a la subvención concedida por la Universidad del País Vasco al Proyecto de Investigación I/UPV 00006.231-H-14035/2001.

Profesora Titular de Psicodiagnóstico de la Infancia y Adolescencia. Colaboradora en el Servicio de Psicología Aplicada de la Facultad de Psicología. Líneas de investigación: Técnicas proyectivas gráficas, Trastornos de imagen corporal y de la conducta alimentaria; Estudios sobre el concepto de la muerte en los niños. Factores de riesgo en adolescentes. Temas de adopción. Facultad de Psicología. Avda. de Tolosa, 70. 20018 San Sebastián. España. Correo Electrónico: ptpmamac@ss.ehu.es. 

Los trastornos del comportamiento alimenticio han ido incrementándose en los últimos años y ampliándose el rango de edad de inicio de los mismos a edades más tempranas. Una percepción distorsionada de la imagen del propio cuerpo, vinculada a una insatisfacción corporal, está en la base de estos trastornos.

Muchas investigaciones han demostrado el papel de los factores socioculturales en el origen de los trastornos alimentarios. La "cultura de la delgadez" (Vandereycken y Meerman, 1984) que se ha instalado en el mundo occidental preconiza cuerpos delgados y con formas poco redondeadas como sinónimos de esbeltez, belleza y poder.

La importancia de este fenómeno entre la población normal, en concreto en adolescentes, ha sido objeto de varias investigaciones (Crisp y Kalucy, 1974; Garner y Garfinkel; Touyz, Beumont, Collins, McCabe y Jupp, 1984) señalando la existencia de una relación entre edad y sobreestimación del tamaño corporal (a menor edad, mayor sobreestimación). Brush (1962) fue la primera autora que sugirió que la distorsión de la imagen corporal, demostrada por una sobreestimación del tamaño, era una característica patognomónica de la anorexia nerviosa. Desde esta fecha muchos estudios han mostrado interés por la importancia que tiene en la génesis de los trastornos alimenticios la distorsión de la imagen corporal (Castro y Toro, 1989; Garner y Garfinkel, 1981; Hsu, 1982; Raich, Torras y Mora, 1997; Rojo y Turón, 1989; Toro, Salamero y Martínez, 1995; Vaz, Salcedo, Suárez y Psicodiagnóstico de la Infancia y Adolescencia y docente en el área clínica. Profesora en el Master de Terapia de Pareja. Líneas de investigación: Técnicas de diagnóstico infantil, Factores de riesgo en adolescentes, trastornos de la conducta alimentaria y de la imagen corporal. Correo electrónico: ptpcrsam@ss.ehu.es 
Alcaina, 1992). Sin embargo, otros estudios sostienen la hipótesis de que los problemas de la imagen corporal son problemas clínicos significativos en los que no siempre existe un trastorno de la alimentación diagnosticable (Fisher y Thompson, 1994; Rosen, 1993). Perpiña (1989) encontró que los límites entre la población clínica y no clínica llegan a difuminarse debido al deseo de las adolescentes de poseer cuerpos cada vez más delgados.

Por todo ello, las investigaciones a este respecto se han centrado en identificar las variables asociadas al desarrollo de alteraciones clínicamente relevantes de la imagen corporal. Reiterados estudios han demostrado que los trastornos de alimentación están asociados a la distorsión e insatisfacción de la imagen corporal. Las variables asociadas a dicha alteración están relacionadas con la edad, el sexo, el índice de masa corporal (IMC), la clase social media-alta, población urbana, la incorporación de la cultura de la delgadez y niveles de ansiedad altos con relación al incremento de peso (Garner y Garfinkel, 1981; Toro, 1988; Perpiña, 1989; Martínez, Toro, Salamero, Blecua y Zaragoza, 1993; Turón, Fernández y Vallejo, 1992; Toro, Salamero y Martínez, 1995). Se ha visto que las alteraciones de la imagen corporal tienen una participación causal en el trastorno, en lugar de ser secundarias a él, lo que les confiere un estatus diferente. Richards, Thompson y Coovert (1990), Hawkins, Fremouw y Clement (1984) confirman que la relación entre disfunción alimentaria y alteración de la imagen corporal (insatisfacción corporal) es unidireccional (son las alteraciones de la imagen corporal las que contribuyen a un trastorno alimentario y no la secuencia inversa).

Según las investigaciones de Mora y Raich (1993) la insatisfacción y la preocupación por el propio cuerpo conducen a una distorsión perceptiva y no a la inversa. Dicha insatisfacción no es un fenómeno estático, sino que se nutre de estímulos ambientales que afectan a la distorsión del tamaño y a la preferencia por la delgadez, activando ansiedad y miedo a la ganancia de peso. 
La ansiedad en sus diversas formas con relación a la comida, el temor a incrementar peso, la obsesión por la delgadez y el perfeccionismo, son variables asociadas y por tanto factores de riesgo. Toro y Vilardell (1987), defienden que la preocupación ansiosa explicaría la relación entre insatisfacción corporal y la distorsión perceptiva. Sostienen que cuando algo preocupa es atendido y observado con ansiedad, facilitando la distorsión de lo percibido. La observación reiterada y ansiosa conduciría a distorsiones, dándose un feedback positivo entre preocupación corporal y sobreestimación.

De acuerdo a estos presupuestos, nuestra investigación tiene por objetivos (a) conocer las relaciones significativas de los siguientes factores de riesgo: edad, sexo, índice de masa corporal, satisfacción corporal, distorsión de la imagen corporal, nivel de ansiedad y obsesión por la delgadez, y (b) en qué medida estas variables son posibles predictores de los trastornos de alimentación.

\section{Metodología}

\section{Participantes}

Participaron 200 adolescentes de 13 años 6 meses a 17 años 11 meses (104 chicos y 96 chicas). Todos pertenecen a un centro educativo privado que solicitó un estudio sobre factores de riesgo en adolescentes de segundo ciclo de Enseñanza Secundaria Obligatoria y de los cursos de Bachiller. El centro está especialmente motivado a implementar programas preventivos con adolescentes habida cuenta del incremento de trastornos alimenticios que han detectado en los últimos 5 años.

\section{Instrumentos}

En la presente investigación se han utilizado los siguientes instrumentos: el Cuestionario de Autoevaluación de Ansiedad Estado/ 
Rasgo (STAI. Spielberger, Gorsuch y Lushene, 1988); el Inventario de Trastornos de la Conducta Alimentaria (EDI-2. Garner, 1998). Eating Attitudes Test (EAT. Garner, Olmstead, Bohr y Garfinkel, 1982; Castro, Toro, Salamero y Guimerá, 1991). Otros dos instrumentos aplicados están en vías de experimentación: la Escala de Satisfacción Corporal (ECS) (Maganto, Cruz, Del Río y Roiz, 2000) y el Test de Siluetas (Maganto, Cruz, Del Río y Roiz, 2000).

La Escala de Satisfacción Corporal consta de 17 ítems y valora el grado de satisfacción que los adolescentes tienen de cada una de las partes de su cuerpo, así como la valoración del mismo tomado en su conjunto. La puntuación de cada una de las partes del cuerpo oscila entre 0 (muy insatisfecho) y 5 (muy satisfecho). Las partes del cuerpo (16 ítems) van integradas en cuatro zonas corporales: Cara (pelo, ojos, nariz y boca), Torso Superior (hombros, pecho, estómago y cintura), Torso Inferior (caderas, nalgas, muslos y piernas) y Otros (tono muscular, piel, altura y peso). La suma de las partes que integran cada zona tiene una puntuación mínima de 0 y máxima de 20. El "Aspecto Físico Global" (AFG), ítem 17, se valora con una puntuación que va desde 1 (muy insatisfecho) a 10 (muy satisfecho) y es la medida de su satisfacción tomando el cuerpo en su conjunto.

El Test de Siluetas (adaptado del Test de Siluetas de Bell, Kirkpatrick y Rinn, 1986) consiste en 8 figuras femeninas que incrementan progresivamente su figura corporal con relación al incremento de peso, y 8 figuras masculinas de similar condición. Se les hacen diversas preguntas sobre las figuras, pero las que interesan en el presente estudio son: "¿qué figura representa tu peso actual?”, y "¿qué figura representa tu peso ideal? o ¿cómo te gustaría ser?".

La primera pregunta permite valorar la distorsión entre el peso real y el peso percibido, y que hemos denominado TS-Distorsión. La segunda pregunta valora la diferencia entre el peso percibido y el peso ideal, es decir, la insatisfacción corporal, y que hemos denominado TS-Insatisfacción. En ambos casos puntuaciones de cero o 
próximas a cero indicarían adecuación o satisfacción con la imagen corporal. Puntuaciones por debajo o sobre cero indicarían el grado en que se da la distorsión o insatisfacción corporal.

\section{Procedimiento}

Las pruebas psicológicas fueron aplicadas en el centro educativo en horas lectivas y en similares condiciones de lugar, tiempo y motivación. Los padres fueron informados previamente del estudio con el objeto de solicitar los permisos pertinentes. Se excluyeron de la muestra los sujetos que no cumplimentaron todos los instrumentos de evaluación.

\section{Resultados y discusión}

Se han realizado análisis descriptivos para conocer el valor de las variables, así como ANOVAs a fin de comprobar las diferencias estadísticas entre las variables estudiadas. Las correlaciones han permitido comprobar el grado de relación entre dichas variables y, finalmente, el análisis de regresión múltiple aporta resultados sobre la capacidad explicativa de los problemas de imagen corporal en los trastornos alimenticios.

Presentamos los Cuadros 1 y 2 que en otras publicaciones han sido objeto de trabajo de investigación, pero las consideramos necesarias para entender los resultados posteriores. 


\section{Cuadro 1}

Media, desviación típica y significatividad de la altura, el peso y del índice de Masa corporal (IMC) por edad y sexco

\begin{tabular}{|c|c|c|c|c|c|c|c|}
\hline \multirow{2}{*}{$\begin{array}{l}\text { Edad } \\
(a \tilde{o}) s)\end{array}$} & & \multicolumn{2}{|c|}{ Altura } & \multicolumn{2}{|c|}{ Peso } & \multicolumn{2}{|c|}{ IMC } \\
\hline & & Varone & Mujeres & Varones & Mujeres & Varones & Mujeres \\
\hline & $M$ & 1.69 & 1.61 & 57.91 & 50.84 & 20.24 & 19.63 \\
\hline 14 & $D E$ & 0.06 & 0.08 & 8.07 & 5.47 & 2.15 & 1.62 \\
\hline Varones $=23$ & $t$ & \multirow{2}{*}{\multicolumn{2}{|c|}{$\begin{array}{r}3.679 \\
.001\end{array}$}} & \multirow{2}{*}{\multicolumn{2}{|c|}{$\begin{array}{r}3.580 \\
.001\end{array}$}} & \multirow{2}{*}{\multicolumn{2}{|c|}{$\begin{array}{r}1.106 \\
.275\end{array}$}} \\
\hline Mujeres $=25$ & $p$ & & & & & & \\
\hline & $M$ & 1.75 & 1.66 & 65.17 & 60.23 & 21.23 & 21.76 \\
\hline 15 & $D E$ & 0.05 & 0.04 & 9.74 & 7.86 & 2.52 & 2.88 \\
\hline Varones $=35$ & $t$ & \multirow{2}{*}{\multicolumn{2}{|c|}{$\begin{array}{r}5.839 \\
.000\end{array}$}} & \multirow{2}{*}{\multicolumn{2}{|c|}{$\begin{array}{r}2.004 \\
.050\end{array}$}} & \multirow{2}{*}{\multicolumn{2}{|c|}{$\begin{array}{r}-.730 \\
.469\end{array}$}} \\
\hline Mujeres $=22$ & $p$ & & & & & & \\
\hline & $M$ & 1.78 & 1.65 & 67.11 & 58.03 & 21.14 & 21.41 \\
\hline 16 & $D E$ & 0.06 & 0.06 & 5.53 & 7.35 & 1.92 & 3.23 \\
\hline Varones $=19$ & $t$ & \multirow{2}{*}{\multicolumn{2}{|c|}{$\begin{array}{r}7.075 \\
.000\end{array}$}} & \multirow{2}{*}{\multicolumn{2}{|c|}{$\begin{array}{r}4.589 \\
.000\end{array}$}} & \multirow{2}{*}{\multicolumn{2}{|c|}{$\begin{array}{r}-.334 \\
.740\end{array}$}} \\
\hline Mujeres $=29$ & $p$ & & & & & & \\
\hline & $M$ & 1.78 & 1.67 & 72.44 & 57.35 & 22.89 & 20.44 \\
\hline 17 & $D E$ & 0.05 & 0.04 & 9.29 & 5.78 & 2.42 & 2.00 \\
\hline Varones $=17$ & $t$ & \multirow{2}{*}{\multicolumn{2}{|c|}{$\begin{array}{r}6.480 \\
000\end{array}$}} & \multicolumn{2}{|c|}{5.995} & \multicolumn{2}{|c|}{3.492} \\
\hline Mujeres $=27$ & $p$ & & & \multicolumn{2}{|c|}{.000} & \multicolumn{2}{|c|}{.001} \\
\hline
\end{tabular}

En el Cuadro 1 se observan diferencias significativas en altura y peso en las edades estudiadas, presentando los chicos mayor peso y estatura. No se crece de forma uniforme con el desarrollo evolutivo. A la edad de 15 años, tanto hombres como mujeres crecen significativamente más que en las otras edades. Las chicas parecen estancarse a partir de esta edad, mientras que los chicos lo hacen un año más tarde.

Con el peso ocurre algo similar. El mayor aumento de peso, tanto en hombres como en mujeres, ocurre de 14 a 15 años, incluso de manera más espectacular en las mujeres. Los chicos incrementan paulatinamente su peso hasta en $15 \mathrm{~kg}$. en cuatro años (de $57.91 \mathrm{a}$ los 14 años a 72.44 a los 17) mientras que las mujeres, a partir del incremento de peso a los 14 años, comienzan a disminuir su peso hasta los 17 (60.23 a los 14 años y 57.35 a los 17). 
La insatisfacción corporal

\section{Cuadro 2}

Diferencias de medias entre hombres y mujeres en la Escala de Satisfacción Corpo$\operatorname{ral}(E S C)$

\begin{tabular}{|c|c|c|c|c|c|c|}
\hline & Partes del $\mathrm{cl}$ & uerpo & $M$ & $D E$ & $t$ & $p$ \\
\hline Cara & $\begin{array}{l}\text { Pelo } \\
\text { Ojos } \\
\text { Nariz } \\
\text { Boca }\end{array}$ & $\begin{array}{l}\text { Varones } \\
\text { Mujeres } \\
\text { Varones } \\
\text { Mujeres } \\
\text { Varones } \\
\text { Mujeres } \\
\text { Varones } \\
\text { Mujeres }\end{array}$ & $\begin{array}{l}3.22 \\
3.24 \\
3.58 \\
3.60 \\
2.68 \\
1.96 \\
3.40 \\
2.83\end{array}$ & $\begin{array}{l}1.36 \\
1.43 \\
1.20 \\
1.39 \\
1.50 \\
1.47 \\
1.26 \\
1.60\end{array}$ & $\begin{array}{r}-0.106 \\
-0.125 \\
3.43 \\
2.82\end{array}$ & $\begin{array}{l}.916 \\
.900 \\
.001 \\
.005\end{array}$ \\
\hline Torso superior & $\begin{array}{l}\text { Hombros } \\
\text { Pecho } \\
\text { Estómago } \\
\text { Cintura }\end{array}$ & $\begin{array}{l}\text { Varones } \\
\text { Mujeres } \\
\text { Varones } \\
\text { Mujeres } \\
\text { Varones } \\
\text { Mujeres } \\
\text { Varones } \\
\text { Mujeres }\end{array}$ & $\begin{array}{l}3.40 \\
2.71 \\
3.01 \\
2.03 \\
3.12 \\
1.84 \\
2.98 \\
1.99\end{array}$ & $\begin{array}{l}1.46 \\
1.49 \\
1.49 \\
1.55 \\
1.46 \\
1.60 \\
1.48 \\
1.53\end{array}$ & $\begin{array}{l}3.34 \\
4.54 \\
5.81 \\
4.63\end{array}$ & $\begin{array}{l}.001 \\
.000 \\
.000 \\
.000\end{array}$ \\
\hline Torso inferior & $\begin{array}{l}\text { Caderas } \\
\text { Nalgas } \\
\text { Muslos } \\
\text { Piernas }\end{array}$ & $\begin{array}{l}\text { Varones } \\
\text { Mujeres } \\
\text { Varones } \\
\text { Mujeres } \\
\text { Varones } \\
\text { Mujeres } \\
\text { Varones } \\
\text { Mujeres }\end{array}$ & $\begin{array}{l}\mathbf{3 . 0 5} \\
\mathbf{1 . 5 2} \\
2.91 \\
1.46 \\
\mathbf{3 . 0 4} \\
\mathbf{1 . 1 7} \\
3.39 \\
1.79\end{array}$ & $\begin{array}{l}1.40 \\
1.48 \\
1.43 \\
1.60 \\
1.49 \\
1.46 \\
1.47 \\
1.90\end{array}$ & $\begin{array}{l}7.46 \\
6.71 \\
8.92 \\
6.62\end{array}$ & $\begin{array}{l}.000 \\
.000 \\
.000 \\
.000\end{array}$ \\
\hline Otros & $\begin{array}{l}\text { Tono muscular } \\
\text { Piel } \\
\text { Altura } \\
\text { Peso }\end{array}$ & $\begin{array}{c}\text { Varones } \\
\text { Mujeres } \\
\text { Varones } \\
\text { Mujeres } \\
\text { Varones } \\
\text { Mujeres } \\
\text { Varones } \\
\text { Mujeres }\end{array}$ & $\begin{array}{l}\mathbf{3 . 5 5} \\
\mathbf{1 . 9 5} \\
3.40 \\
2.70 \\
3.88 \\
2.44 \\
2.88 \\
1.80\end{array}$ & $\begin{array}{l}1.36 \\
1.43 \\
1.40 \\
1.55 \\
1.39 \\
1.74 \\
1.46 \\
1.80\end{array}$ & $\begin{array}{l}8.05 \\
3.35 \\
4.16\end{array}$ & $\begin{array}{l}.000 \\
.001 \\
.000 \\
.000\end{array}$ \\
\hline \multicolumn{2}{|c|}{$\begin{array}{l}\text { Aspecto físico global } \\
\text { (De } 0 \text { a } 10 \text { ) }\end{array}$} & $\begin{array}{l}\text { Varones } \\
\text { Mujeres }\end{array}$ & $\begin{array}{l}7.47 \\
5.95\end{array}$ & $\begin{array}{l}1.24 \\
1.85\end{array}$ & 6.36 & .000 \\
\hline
\end{tabular}


Con respecto al IMC, no hay diferencias significativas entre hombres y mujeres, excepto a los 15 años. Esto es debido a que las mujeres disminuyen su IMC durante su desarrollo evolutivo, debido a la reducción del peso físico. Este podría ser un indicador de la preocupación por el cuerpo y por el deseo de estar delgadas a medida que se incremente la edad.

Los datos del Cuadro 2 indican que las mujeres están más insatisfechas que los hombres con todas las partes del cuerpo escrutadas, excepto en el pelo y los ojos. La cara es la parte del cuerpo de la que más satisfechas están las mujeres. Estos resultados muestran que estas son las partes que a las adolescentes les preocupa menos, quizás porque el estereotipo de imagen corporal que la sociedad marca es mucho más concreto y específico en las partes del tronco (torso superior e inferior) mientras que los rasgos de la cara tienen un abanico más amplio de posibilidades que se valoran socialmente de forma positiva.

Con respecto al torso superior, los hombros conforman la parte más valorada y la cintura es la que más descontento produce a ambos. Las mayores diferencias entre chicos y chicas están en el estómago, parte del cuerpo de especial insatisfacción para las mujeres.

En el torso inferior es donde se observan más diferencias entre hombres y mujeres. Mientras que los hombres tienen una puntuación media de 3 ó más, las mujeres no alcanzan una puntuación de 2. Las partes que componen el torso inferior son las que más insatisfacción crean a las mujeres, especialmente los muslos $(M=1.17)$, las nalgas $(M=1.43)$ y las caderas $(M=1.52)$. Son por tanto las zonas de mayor preocupación e insatisfacción para ellas.

Los hombres están especialmente satisfechos de su altura, tono muscular y piel. Son partes del cuerpo que hemos integrado en el apartado "Otros" y en donde ellos obtienen puntuaciones más altas. 
La puntuación del peso baja en ambos, hombres $(M=2.88)$ y mujeres $(M=1.80)$. Esto prueba la preocupación e insatisfacción en ambos sexos por el peso corporal.

En síntesis, lo que más les disgusta a las mujeres de su cuerpo analizándolo parte por parte, son: muslos $(M=1.17)$, nalgas $(M=$ $1.46)$, caderas $(M=1.52)$, piernas $(M=1.79)$, peso $(M=1.80)$ y estómago $(M=1.84)$. Lo que menos les gusta a los hombres es: la nariz $(M=2.68)$, el peso $(M=2.88)$, las nalgas $(M=2.91)$ y la cintura $(M=2.98)$.

La puntuación del Aspecto Físico Global es más alta que el sumatorio de las zonas corporales aisladamente tanto en chicos como en chicas. Es decir, parece que los adolescentes se valoran más positivamente observando el conjunto de todo su cuerpo, pero son mucho más críticos si se analizan parte por parte. En cualquier caso, también en el Aspecto Físico Global las chicas se puntúan alrededor de dos puntos por debajo de los chicos $(M=5.95$ y $M=7.47$ respectivamente, $t=6.36, p=.000$ ).

Para conocer si las diferencias de puntuación en las zonas corporales dependían de la edad de los participantes, del sexo y/o de la interacción de ambas variables, se procedió a realizar los análisis de varianza. La suma de las puntuaciones de las cuatro partes del cuerpo que constituyen una zona corporal puede oscilar entre 0 (muy insatisfecho) a 20 (muy satisfecho). Los resultados se exponen en las figuras 1 a 5 . 


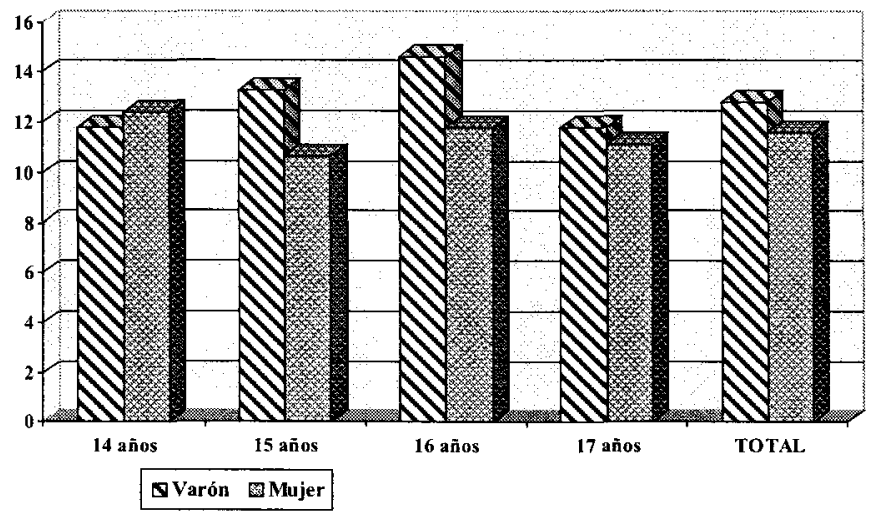

Figura 1. Satisfacción con la cara (de 0 a 20 ) según edad y sexo.

La Figura 1 muestra el grado de satisfacción general que tanto hombres como mujeres tienen hacia su cara, obteniendo ambos puntuaciones por encima de 10. La única fuente de variación es el sexo, $\mathrm{F}(1.91)=5.92$ y $p=.016$. No existen diferencias significativas a lo largo de las edades $F(3.191)=1.57, p=.196$, aunque las mujeres están más insatisfechas de su cara a los 14 años y los hombres más satisfechos a los 15 años. Tampoco existen diferencias significativas en la interacción edad y $\operatorname{sexo} F(3.191)=2.06, p=.107$.

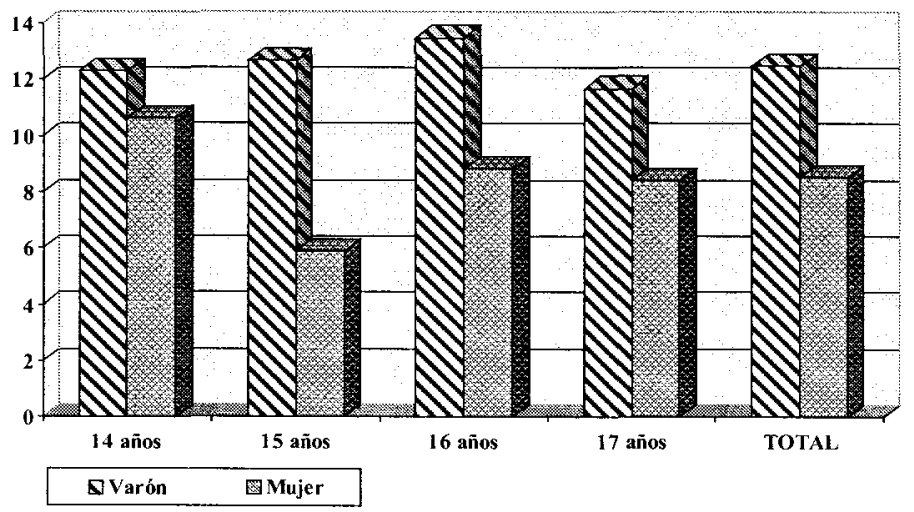

Figura 2. Satisfacción con el torso superior (de 0 a 20) según edad y sexo. 
En la Figura 2 se aprecia que las diferencias de sexo son evidentes $\mathrm{F}(1.91)=36.39$ y $p=.000 ; M=12.47$ en hombres y $M=$ 8.55 en mujeres. Los resultados del análisis de la ANOVA por edades $F(3.191)=2.32$ y $p=.077$ y la interacción de edad y sexo $F$ $(3.191)=2.75$ y $p=.044$, sugieren que la valoración que los adolescentes hacen del torso superior sólo tendencialmente depende de la edad. Ésta se convierte en una fuente de variación en interacción con el sexo. No obstante, la edad de mayor insatisfacción en las mujeres es la de los 15 años ( $M=5.91$ en mujeres y $M=12.68$ en hombres) y la de mayor satisfacción para los hombres es la de los 16 años $(M$ $=13.47$ en hombres y $M=8.81$ en mujeres).

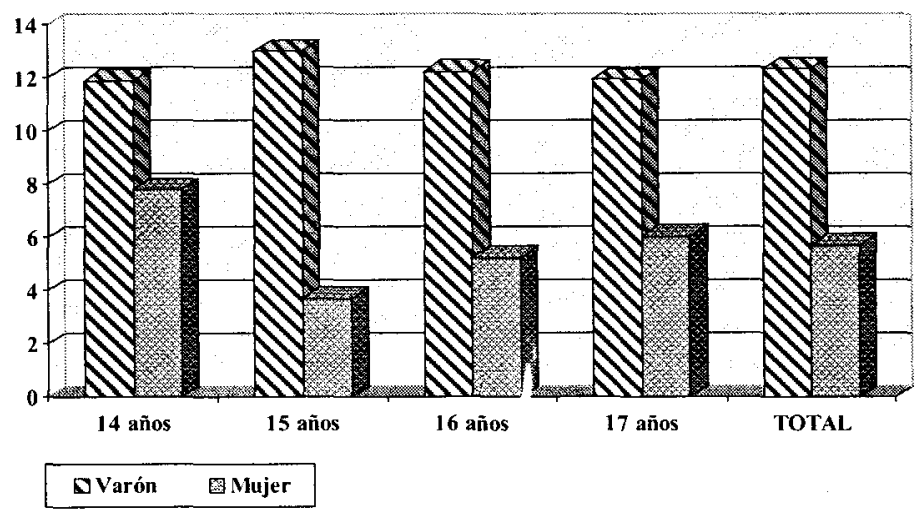

Figura 3. Satisfacción con el Torso Inferior (de 0 a 20) por edad y sexo

La Figura 3 muestra las diferencias de satisfacción corporal que existen entre hombres $(M=12.33)$ y mujeres $(M=5.71)$ en el torso inferior $F(1.91)=87.29$ y $p=.000$. Esto se mantiene independientemente de la edad, $F(3.191)=083, p=.474$, pero en la interacción de ambas variables las diferencias son significativas $F(3.191)=$ 2.58 y $p=.055$. De nuevo la edad con puntuaciones inferiores para las mujeres es la de los 15 años, donde las puntuaciones alcanzan las mayores diferencias en función del sexo $(M=13$ para hombres y 3.73 para mujeres). Esto indica que los hombres se encuentran satis- 
fechos con su torso inferior con puntuaciones por encima de 12 , y las mujeres se encuentran muy a disgusto y muy insatisfechas con todas las partes que forman el torso inferior: caderas, nalgas, muslos y piernas. Estas puntuaciones confirman que esta zona es especialmente sensible para la valoración personal de la mujer y más a los 15 años.

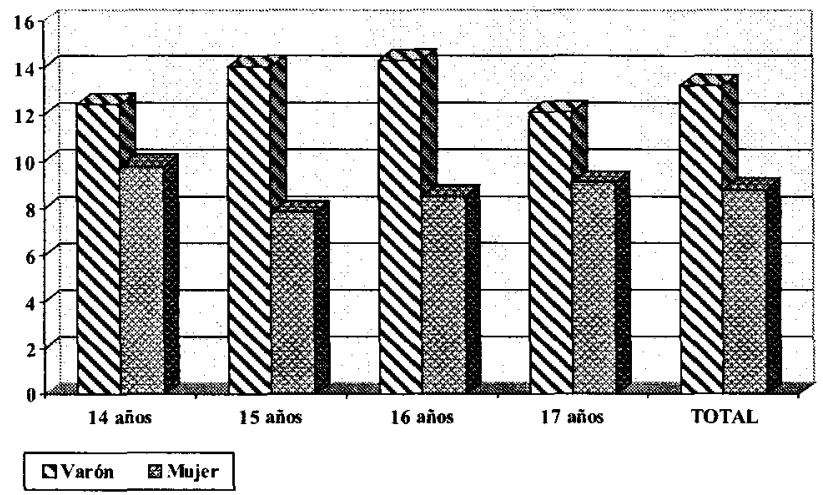

Figura 4. Satisfacción con Otras partes del cuerpo (de 0 a 20) por edad y sexo

El ANOVA correspondiente a la Figura 4 indica que sólo el sexo es una fuente de variación con relación a esta zona corporal denominada "otras" partes del cuerpo $(M=13.22$ para hombres y $M=8.80$ para mujeres; $F(1.91)=50.49$ y $p=.000)$. La edad no influye en la satisfacción presentada en esta zona corporal, $F(3.191)=.26, p=$ .851. Tendencialmente se observa que la interacción de la edad y el sexo hace variar las puntuaciones obtenidas $F(1.91)=2.24$ y $p=$ .085. De nuevo la insatisfacción es mayor en las mujeres a lo largo de las edades. Los hombres, sin embargo, se encuentran satisfechos, incluso valoran más esta zona $(M=13.22)$ que las otras zonas corporales: cara $(M=12.82)$, torso superior $(M=12.47)$ y torso inferior $(M=12.33)$. 


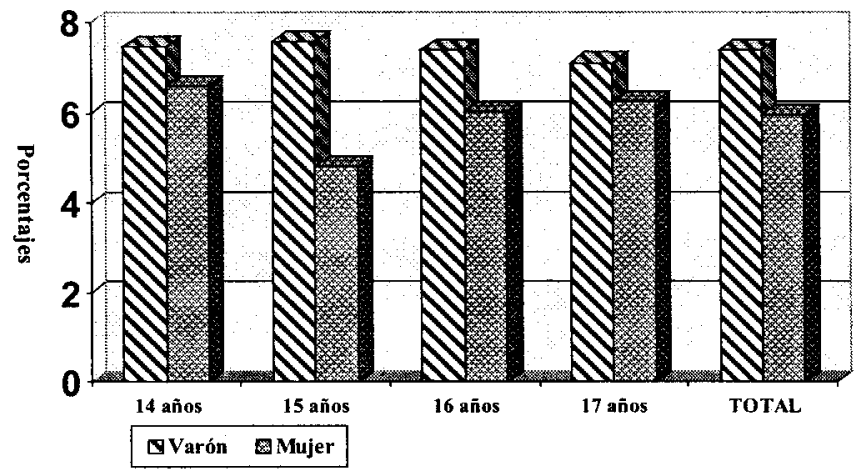

Figura 5. Aspecto Físico Global (de 0 a 10) por edad y sexo.

La Figura 5, en consecuencia con el análisis de varianza del Cuadro 7, demuestra, como ocurre a lo largo de la Escala de Satisfacción Corporal, que los chicos puntúan por encima de las chicas a lo largo de todas las edades. El análisis de varianza indica que la puntuación del Aspecto Físico Global depende de la edad $F(3.191)=2.61$ y $p=$ .053 , del sexo $F(1.91)=44.02$ y $p=.000$, y de la interacción de la edad y sexo $(M=7.40$ para chicos y $M=5.95$ para chicas, $F(1.91)=$ 4.48 y $p=.005$. Las mayores diferencias por edad se encuentran a los 15 años $(M=7.59$ para chicos y $M=4.81$ para chicas) en el que las chicas incrementan significativamente de forma sensible su peso físico.

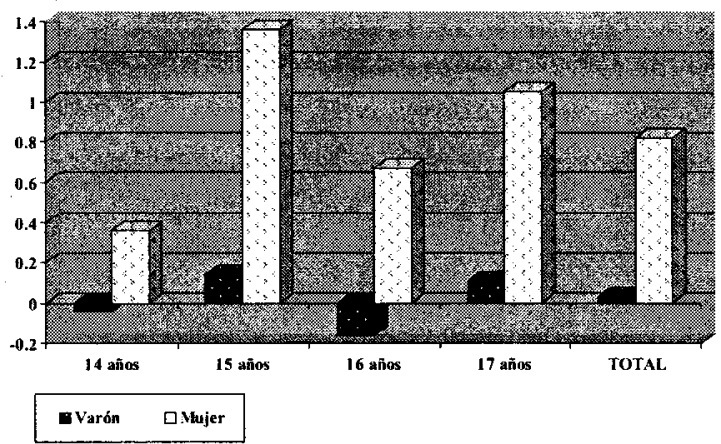

Figura 6. TS-Insatisfacción por edad y sexo. Puntuación positiva: deseo de estar más detgado. Puntuación negativa: deseo de estar más gordo. 
Los resultados de la Figura 6 son contundentes. Los chicos $(M=$ .038) como indican los resultados próximos a cero, están más satisfechos que las chicas $(M=0.821)$. La ANOVA indica que el edad es una fuente importante de variación con relación a la insatisfacción $F$ $(3.191)=3.93$ y $p=.009)$ y también el sexo $F(1.91)=34.73$ y $p=$ .000. Los chicos que están insatisfechos desean estar más gruesos de lo que se perciben, mientras que las chicas, a todas las edades, desean estar más delgadas. La edad de 15 años es en la que más insatisfechas están las mujeres, corroborando de nuevo ser una edad especialmente vulnerable a los problemas de imagen corporal. A esta edad, 15-16 años, desearían estar una figura menos gruesas de lo que se perciben.

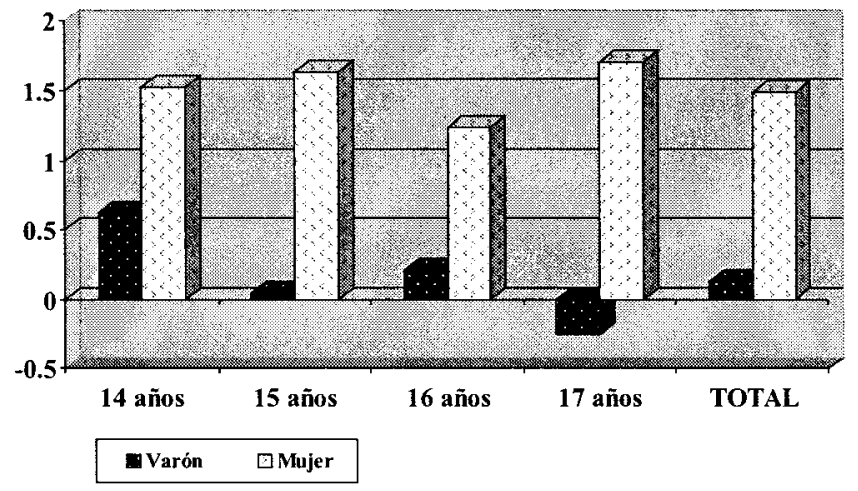

Figura 7. TS-Distorsión de la Imagen Corporal por edad y sexo. Puntuación positiva: verse más gordo. Puntuación negativa: verse más delgado.

La variable TS-Distorsión ha sido creada también a partir de los resultados del Test de Siluetas. Se ha calculado el valor de la resta entre qué figura representa su peso actual y el IMC real de cada sujeto. Los valores próximos a cero indican que no existe distorsión, mientras que los valores que se alejan de cero indican el mayor o menor grado de distorsión y la dirección que sigue la misma. 
El análisis de varianza con relación al sexo $F(1.91)=92.90$ y $p$ $=.000$ demuestra que los hombres $(M=0.135)$ en todas las edades tienden a percibirse significativamente con más ajuste a su IMC que las mujeres $(M=1.49)$. La edad por sí misma no es una fuente de variación con relación a la distorsión $F(3.191)=1.32, p=.268$. Las relaciones significativas en la interacción edad y sexo $F(3,191)=$ 2.98 y $p=.032$ provienen más de las diferencias de sexo que de las diferencias de edad. La distorsión de las chicas muestra que se ven más gruesas de lo que en realidad están, lo que puede explicar los resultados de la insatisfacción corporal que mostraba la figura anterior.

El Cuadro 3 muestra las altas correlaciones existentes entre las variables estudiadas. La subescala EDI-Insatisfacción muestra altas correlaciones con la subescala EDI-Obsesión por Ia delgadez ( $r=$ $.74, p<.001)$ lo que confirma que son un único factor de la escala. Presenta, asimismo, altas correlaciones con el EAT $(r=.59, p<$ $.001)$ lo que parece indicar que miden aspectos similares. También las correlaciones con la ansiedad rasgo $(r=.31, p<.001)$ sugieren que la obsesión por la delgadez se presenta con alta frecuencia en sujetos con importantes niveles de ansiedad y con ansiedad relacionada con el cuerpo. Además, la correlación negativa entre EDI-Insatisfacción y el Aspecto Físico Global de la Escala de Satisfacción Corporal $(r=-.66, p<.001)$ parece indicar que la Escala de Satisfacción Corporal tiene validez de contenido, aspecto que está siendo investigado recientemente. Otras dos correlaciones importantes se establecen entre las variables EDI-Insatisfacción, la TS-Insatisfacción $(r=.60, p<$ $.001)$ y la TS-Distorsión $(r=.40, p<.001)$ mostrando la estrecha relación entre las mismas y posiblemente el hecho de estar midiendo un factor similar. El peso real (IMC) presenta correlaciones inferiores a las anteriores $(r=.21, p<.01)$, lo cual demuestra la importancia del peso percibido sobre el peso real para la ocurrencia de los trastornos de alimentación. 


\section{Cuadro 3}

Correlaciones entre EDI-2, Ansiedad, EAT, Escala de Satisfacción Corporal, IMC, Insatisfacción y Distorsión $(N=197)$

\begin{tabular}{|c|c|c|c|c|c|c|c|c|}
\hline & $\begin{array}{c}\text { EDI } \\
\text { Insatisfacción }\end{array}$ & $\begin{array}{c}\text { EDI } \\
\text { Obsesión } \\
\text { por } \\
\text { delgadez }\end{array}$ & $\begin{array}{l}\text { Ansiedad } \\
\text { Estado }\end{array}$ & $\begin{array}{c}\text { Ansiedad } \\
\text { Rasgo }\end{array}$ & EAT & $\begin{array}{c}\text { Aspecto } \\
\text { Físico } \\
\text { Global }\end{array}$ & IMC & $\begin{array}{c}\text { TS } \\
\text { Insatisfacción }\end{array}$ \\
\hline \multicolumn{9}{|l|}{$\begin{array}{l}\text { EDI } \\
\text { Insatisfacción }\end{array}$} \\
\hline $\begin{array}{l}\text { EDI Obsesión } \\
\text { por delgadez }\end{array}$ & $.744 * * *$ & & & & & & & \\
\hline $\begin{array}{l}\text { Ansiedad } \\
\text { Estado }\end{array}$ & $.164 *$ & $.222 * *$ & & & & & & \\
\hline $\begin{array}{l}\text { Ansiedad } \\
\text { Rasgo }\end{array}$ & $.317^{* * *}$ & $.709 * * *$ & $.709 * * *$ & & & & & \\
\hline EAT & $.595^{* * *}$ & $.750^{* * *}$ & $.193 * *$ & $.328^{* * *}$ & & & & \\
\hline $\begin{array}{l}\text { Aspecto Físico } \\
\text { Global }\end{array}$ & $-668^{* * *}$ & $-.495^{* * * *}$ & $-.200^{* *}$ & $-.338 * * *$ & $-.418 * * *$ & & & \\
\hline IMC & $.216^{* *}$ & .111 & $-.160^{*}$ & $-.169^{*}$ & -.043 & $-.211 * *$ & & \\
\hline TS-Insatisfacción & $.608 * * *$ & $.536^{* * *}$ & .090 & $.168^{*}$ & $.393^{* * *}$ & $-.507 * * *$ & $.439 * * *$ & \\
\hline TS-Distorsión & $403^{* * *}$ & $.347^{* * *}$ & .138 & $.239 * * *$ & $.347 * * *$ & $-.294 * * *$ & $-.401 * * *$ & $497^{* * *}$ \\
\hline
\end{tabular}

Nota. ${ }^{* * *} p<.001 \quad * * p<.01 \quad * p<.05$ 
La subescala EDI-Obsesión por la delgadez presenta las correlaciones más altas con el EAT $(r=.75, p<.001)$-lo que parece indicar que es el mismo factor- y con la ansiedad rasgo $(r=.70, p<$ .001 ), lo que sugiere la asociación de ambas variables en los trastornos alimenticios. También esta subescala presenta correlaciones altas y negativas con el Aspecto Físico Global $(r=-.49, p<.001)$ confirmando que a menor satisfacción corporal mayor obsesión por adelgazar. Las correlaciones con TS-Insatisfacción $(r=.53, p<.001)$ y con TS-Distorsión $(r=.34, p<.001)$ corroboran una vez más la interrelación de los trastornos de la imagen corporal por insatisfacción y distorsión y la obsesión por la pérdida de peso.

La ansiedad estado solamente presenta altas correlaciones con la ansiedad rasgo $(r=.70, p<.001)$ y moderadas con el EAT $(r=.19$, $p<.01)$ y con el Aspecto Físico Global de forma negativa $(r=-.20$, $p<.01)$. Algo similar ocurre con la ansiedad estado pero con correlaciones más altas. Con el EAT $(r=.32, p<.001)$ van en sentido positivo y con el Aspecto Físico Global en sentido negativo. Parece confirmarse que a mayor insatisfacción, se da una mayor ansiedad y una mayor probabilidad de padecer un trastorno de conducta alimentaria.

Las altas correlaciones del EAT con el Aspecto Físico Global ( $r$ $=-.41, p<.001)$, con TS-Insatisfacción $(r=.39, p<.001)$ y con TSDistorsión confirma la relación entre estas variables: distorsión, insatisfacción y problemas en la alimentación.

El Aspecto Físico Global, como se ha señalado, presenta altas correlaciones con las variables estudiadas y con las variables TS-Insatisfacción $(r=-.50, p<.001)$ y con TS-Distorsión $(r=-.29, p<$ $.001)$ lo que parece apuntar a que el Aspecto Físico Global valora conjuntamente la distorsión y la insatisfacción corporal. Estos resultados sugieren que la Escala diseñada para medir el grado de satisfacción corporal tiene un soporte empírico para hipotetizar su validez de contenido que está siendo investigada en estos momentos. Finalmente las 
altas correlaciones entre las variables TS-Insatisfacción y TS-Distorsión corroboran la relación entre estos aspectos de la imagen corporal.

La fuerza de las correlaciones nos lleva a plantearnos cuáles de estas variables tienen mayor valor predictivo en los trastornos de alimentación y por tanto, qué variables pueden ser consideradas indicadores de riesgo. En el Cuadro 4 se presentan los resultados del análisis de regresión, tomando como variable dependiente la puntuación del EAT e incluyendo en dicho análisis la edad y sexo de los sujetos. Se excluyeron las subescalas del EDI por las altas correlaciones que presentaron y por ser otra prueba para evaluar trastornos alimentarios.

\section{Cuadro 4}

Análisis de regresión múltiple lineal para variables predictoras en el EAT

\begin{tabular}{|l|c|c|c|c|c|}
\hline & $\begin{array}{c}R \\
\text { cuadrado }\end{array}$ & $\begin{array}{c}R \text { cuadrado } \\
\text { corregida }\end{array}$ & Beta & $t$ & $p$ \\
\hline Aspecto físico global & .173 & .168 & -.164 & -.2 .125 & .035 \\
TS-distorsión & .230 & .222 & .193 & 2.773 & .006 \\
Ansiedad de rasgo & .255 & .243 & .297 & 3.627 & .000 \\
Edad & .281 & .266 & .193 & 2.467 & .015 \\
TS-Insatisfacción & .300 & .281 & .171 & 2.261 & .025 \\
\hline
\end{tabular}

Cinco variables entran a formar parte de la predicción de las puntuaciones del EAT. En primer lugar el Aspecto físico Global con un $16,8 \%$ de explicación de la varianza. En segundo lugar se incluye en la ecuación de regresión la variable TS-Distorsión de la propia imagen corporal, que añade un $6 \%$ aproximadamente a la explicación de la varianza. La ansiedad rasgo, como ya quedó patente en el Cuadro de correlaciones, está fuertemente asociada al EAT siendo la tercera variable que forma parte de la predicción de las puntuaciones obtenidas. La edad y la significatividad positiva con el EAT sugieren que a medida que los chicos y chicas aumentan en edad también aumenta la probabilidad de obtener puntuaciones más altas en el EAT, 
lo que a su vez indica que existe mayor riesgo de presentar trastornos de la conducta alimentaria. La TS-Insatisfacción agrega un $2 \%$ a la varianza explicada por las otras variables, que explica entre todas ellas un $28 \%$ de la varianza.

Por lo tanto, bajas puntuaciones en el Aspecto Físico Global, altas puntuaciones en TS-Distorsión, ansiedad rasgo y TS-Distorsión juntamente con el incremento de la edad, se convierten en variables predictoras de posibles trastornos de alimentación.

El hecho de que la variable sexo no entre a formar parte del análisis de regresión múltiple tiene, a nuestro juicio, la siguiente explicación. Cada una de las variables estudiadas presenta diferencias significativas en función del sexo, por lo que el análisis de regresión lo desecha como variable aislada y predictora, al estar comprendido en las variables obtenidas como explicativas predictoras.

\section{Conclusiones}

\section{Índice de Masa Corporal (IMC)}

Las chicas decrecen en IMC durante su desarrollo, debido a la pérdida de peso entre los 14 y los 17 años. Éste es un indicador de su preocupación acerca de su cuerpo y del evidente deseo de estar delgadas a medida que se incrementa la edad y el desarrollo biológico y, consecuentemente, se incrementa su masa corporal.

\section{Escala De Satisfacción Corporal (ESC)}

Atendiendo a la Escala de Satisfacción Corporal, nuestros resultados muestran que las adolescentes están más satisfechas con su pelo, ojos y cara, posiblemente porque los estereotipos sociales son más permisivos con estas partes del cuerpo, pero más exigentes y precisos con las partes que componen el torso superior e inferior. 
La baja puntuación en relación al peso en dicha Escala, confirma la preocupación que los adolescentes de ambos sexos sienten en relación a su peso. Los chicos están más satisfechos con las partes del Torso Superior que las chicas, pero las partes del cuerpo que ocasionan especial insatisfacción en las mujeres son las del torso inferior: caderas, nalga, muslos incluyendo piernas y vientre. Las diferencias significativas y las bajas puntuaciones confirman que ésta es una parte especialmente sensible para las adolescentes, especialmente a la edad de 15 años, cuando el desarrollo se incrementa de forma importante. El hecho de que el Aspecto físico Global obtenga puntuaciones superiores a la suma de las partes consideras aisladamente, indica que los adolescentes de ambos sexos se valoran más positivamente cuando observan el cuerpo tomado en su conjunto, pero son mucho más críticos analizando cada parte por separado. Esta valoración incluye también otros aspectos que no pueden ser contemplados en el análisis pormenorizado de cada parte, como son la forma de mirar, las proporciones, el modo de moverse, etc. Ambos aspectos de la Escala son, por consiguiente, una buena medida de la satisfacción corporal.

Por otra parte, las altas y significativas correlaciones con la subescala del EDI- insatisfacción confirma la validez convergente del instrumento que está siendo investigado en muestras más amplias.

\section{El Test de Siluetas: TS-Distorsión y TS-Insatisfacción}

Uno de los resultados que se repiten de forma insistente es que las chicas están más insatisfechas que los chicos y que obtienen mayores niveles de distorsión de su imagen corporal, especialmente a los 15 años. Esto de nuevo corrobora que las edades de 15-16 años son particularmente vulnerables a los problemas de imagen corporal. A estas edades las chicas desean estar bastante más delgadas de lo que están, siendo las diferencias estadísticamente significativas en relación al sexo y a la edad. Posiblemente la distorsión, es decir, percibirse 
más gordas de los que realmente son, provenga de la insatisfacción corporal.

De nuevo las correlaciones con EDI-Insatisfacción y con EDIObsesión por la delgadez corroboran la validez convergente de este instrumento utilizado a nivel experimental, pero que está siendo objeto de estandarización en muestras interculturales.

Las correlaciones observadas entre Ansiedad, el Aspecto Físico Global y el EAT indican que a mayor ansiedad se da menor satisfacción corporal global y por tanto la probabilidad de padecer un trastorno alimentario aumenta. Por otra parte, las correlaciones entre EAT, el Aspecto Físico Global, las puntuaciones de TS-Distorsión y TS-Insatisfacción, confirman la relación entre problemas alimenticios y las variables de insatisfacción y distorsión.

El análisis de regresión llevado a cabo muestra que las puntuaciones bajas en el Aspecto Físico Global y altas puntuaciones en TSDistorsión, Ansiedad Rasgo y TS-Insatisfacción, juntamente con la edad son factores de riesgo o predictivos de padecer un trastorno alimentario.

No obstante, el bajo porcentaje de la varianza explicada (28\%) indica que es necesario llevar a cabo más investigaciones, incluyendo otras variables clínicas, a fin de predecir, y, por consiguiente, prevenir los trastornos alimenticios en adolescentes.

\section{Referencias}

Altabe, M. y Thompson, J. K. (1992) Size estimation versus figural ratings of body image disturbance: Relation to body dissatisfaction eating dysfunction. International Journal of Eating Disorders, 11, 4, 397-402. 
Andersen, A. E. y Di Domenico, K. (1992). Diet versus shape content popular male and female magazines: A dose-response relationship to the incidence of eating disorders? International Journal of Eating Disorders, 11, 3, 283-287.

Bell, C., Kirkpatrick, W y Rinn, R. C. (1986). Body image of anorexic, obese and normal females. Journal of Clinical Psychology, 42, 431-439.

Brush, H. (1962). Perceptual and conceptual disturbances in anorexia nervosa. Psychosomatic Medicine, 24, 187-194.

Cash, T. F. y Henry, P. (1995). Women's body images: The results of a national survey in the USA. Sex Roles, 33, 19-28.

Castro, J. y Toro, J. (1989). Anorexia mental: Aproximación Psicosocial. Cuadernos de Medicina Psicosomática y sexual, 9, 37-42.

Castro, J., Toro, J., Salamero, M. y Guimerá, E. (1991): The Eating Attitudes Test: Validation of Spanish Version. Evaluación Psicológica / Psychological Assessment, 7 (2): 175-190.

Cooper, P. J. y Goodyer, I. (1997). Prevalence and significance of weight and shape concerns in girls aged 11-16 years. British Journal of Psychiatry, 171, 5422-544.

Crisp, A. H. y Kalucy, R. S. (1974). Aspects of the perceptual disorder in anorexia nervosa. British Journal of Medical Psychology, 47, 349-361.

Fisher, E. y Thompson, J. K. (1994). A comparative evaluation of cognitive-behavioral therapy (CBT) versus exercise theray (ET) for the treatment of body image disturbance: Preliminary findings. Behavior Modification, 19, 143-163.

Garner, D. M. (1998) Inventario de Trastornos de la Conducta alimentaria. Madrid. TEA Ediciones.

Garner, D. M. y Garfinkel, P. E. (1981). Body image in anorexia nervosa: measurements, theory and clinical implications. International Journal of Psychiatry in Medicine, 11, 263-284.

Garner, D. M. y Olmstead, M. P. (1984). The Eating Disorders Inventory Manual. Odesa, Florida. Psychological Assessment Resources. 
Garner, D. M., Olmstead, M. P., Bhor, y Garfinkel, P. E. (1982). The Eating Attitudes Test: Psychometric features and clinical correlates. Psychological Medicine, 12, 871-878.

Gracia de, M., Marcó, M., Fernández, M. y Juan, J. (1999). Autoconcepto físico, modelo estético e imagen corporal en una muestra de adolescentes. Psiquis, 20, (1), 15-26.

Hawkins, R. C., Fremouw, W. I. y Clement, P. (1984). The BingePurge syndrome: Diagnosis, Treatment and Research. Springer: New York.

Hsu, L. K. (1982). Is there a body image disturbance in anorexia nervosa? Journal of Nervous and Mental Disease, 5, 305-307.

Maganto, C. y Cruz, S. (2000). La imagen corporal y los trastornos alimenticios: una cuestión de género. Cuadernos de Psiquiatría y Psicoterapia Infantil, 30, 45-59.

Maganto, C., Del Río, A. y Roiz, O. (2000). Factores de riesgo de trastornos alimenticios. Ponencia presentada en el I Congreso Europeo de Psicoterapia: La Psicoterapia en una Europa Unificada. Barcelona, España.

Maganto, C., Cruz, S., Del Río, A. Y Roiz, O. (2000). Escala de Satisfacción Corporal (ESC). Material no publicado.

Maganto, C., Cruz, S., Del Río, A. y Roiz, O. (2000). Test de Siluetas. (Adaptado de Bell. Kirkpatrick y Rin, 1986). Material no publicado

Martínez, E., Toro, J., Salamero, M., Blecua, J. y Zaragoza, M. (1993). Influencias socioculturales sobre las actitudes y conductas femeninas relacionadas con el cuerpo y la alimentación. $R e$ vista de Psiquiatría de la Facultad de Medicina de Barcelona, (20), 2, 51-65.

Mora, M. y Raich, R. M. (1992). Diferentes aproximaciones teóricas a las alteraciones de la imagen corporal. Cuadernos de Medicina Psicosomática, 23, 55-64.

Perpiña, C. (1989) Hábitos alimenticios, peso e imagen corporal. Revista de la Facultad de Medicina de Barcelona, 6, 303-312.

Raich, R. M., Torras, J. y Figueras, M. (1996). Estudio de la imagen 
corporal y su relación con el deporte en una muestra de estudiantes universitarios. Análisis y Modificación de conducta, 22, (85), 603-626.

Raich, R. M., Torras, J. y Mora, M. (1997). Análisis de las variables influyentes en la aparición de alteraciones de la conducta alimentaria. Psicología, 5, 1, 55-70.

Richards, K. J., Thompson, J. K. y Coovert, M. (1990). Development of body image and eating disturbance. Manuscrito no publicado. Rojo, E. y Turón, V. (1989). Imagen corporal y anorexia nerviosa. Revista de Psiquiatría de la Facultad de Medicina de Barcelona, 16, (6), 313-321.

Rosen, J. C. (1993). Body image disorder: Definition, development and contribution to eating disorders. En J. H. Crowther, S. E. Hobfoll, M. A. P. Stephens y D. L. Tennebaum (Eds.), The etiology of bulimia: The individual and family context. Washington, DC: Hemisphere Publishers.

Royo, T. (1989). Factores socioculturales en la etiología de la anorexia nerviosa. Psicologemas, 3 (5), 115-127.

Spielberger, C. D., Gorsuch, R. L. y Lushene, R. E. (1988). Cuestionario de Ansiedad Estado-Rasgo (STAI). Madrid. TEA Ediciones.

Sunday, S. R., Halmy, K. A., Werdann, L. y Levey, C. (1992). Comparison of body size estimation and eating disorder inventory scores in anorexia and bulimia patients with obese, and restrained and unrestrained controls. International Journal of Eating Disorders, 11, 2, 133-149.

Thompson, J. K. y Thompson, C. M. (1986). Body size distortion and self-esteem in asymptomatic, normal eight males and females. International Journal of Eating Disorders, 6, 1061-1068.

Tiggemann, M. y Pickering, A. S. (1996) Role of television in adolescent women's body dissatisfaction and drive for thinness. Journal of Eating Disorders, 20, 193-202.

Tiggemann, M., Winefield, H. R., Winefiekd, A. H. y Goldney, R. D. (1994). Gender differences in the psychological correlates of body-weight in young adults. Psychology and Health, 9, 345-351. 
Toro, J. y Vilardell, J. (1987). Anorexia Nerviosa. Barcelona. Martínez Roca.

Toro, J. y Vilardell, J. (1987). Anorexia Nerviosa. Barcelona. Martínez Roca.

Toro, J. (1988). Factores socioculturales en los trastornos de la ingesta. Anuario de Psicología, 38, 25-47.

Toro, J., Salamero, M. y Martínez, E. (1995). Evaluación de las influencias socioculturales sobre el modelo estético corporal en la anorexia nerviosa. Revista de Psiquiatría de la Facultad de Medicina de Barcelona, 22, 205-214.

Touyz, S. W., Beumont, P. J., Collins, J. K., McCabe, M. y Jupp, J. (1984). Body shape perception and its disturbance in anorexia nervosa. British Journal of Psychiatry, 144, 167-171.

Turón, J., Fernández, F. y Vallejo, J. (1992). Anorexia nerviosa: características demográficas y clínicas en 107 casos. Revista de Psiquiatría de la Facultad de Medicina de Barcelona, 19 (1), 19-25.

Vandereycken, W. y Meerman, R. (1984). Anorexia nervosa: Is prevention possible?. International Journal of Psychiatry in Medici$n e, 3,15-24$.

Vaz, F. J., Salcedo, M. S., Suarez, F. y Alcaina, T. (1992). Factores socioculturales en la imagen corporal en la mujer. Psiquiatría Pública, (4), 1, 32-37.

Wiseman, C. V., Gray, J. J., Mosimann, J. E. y Ahrens, A, H. (1992). Cultural expectations of thinness in women: An update. International Journal of Eating Disorders, 11, 85-89. 